\title{
An adaptive learning approach for no-wait flowshop scheduling problems to minimize makespan
}

\author{
Orhan Engin * $^{*}$ \\ Department of Industrial Engineering, \\ Faculty of Engineering and Architecture, \\ University of Selçuk, Konya, 42079 Turkey \\ E-mail:orhanengin@yahoo.com \\ Cengiz Günaydın \\ Department of Industrial Engineering, \\ Faculty of Engineering and Architecture, \\ University of Selçuk, Konya, 42079 Turkey \\ www.selcuk.edu.tr
}

Received: $10 / 03 / 2011$

Accepted: 02 / $05 / 2011$

\begin{abstract}
No-wait flowshop scheduling problem (NW-FSSP) with the objective to minimize the makespan is an important sequencing problem in the production plans and applications of no-wait flowshops can be found in several industries. In a NW-FSSP, jobs are not allowed to wait between two successive machines. The NW-FSSPs are addressed to minimize makespan and the NW-FSSP is known as a NP- Hard problem. In this study, Agarwal et al.'s ${ }^{1}$ adaptive learning approach (ALA) is improvement for NW-FSSPs. Improvements in adaptive learning approach is similar to neural-network training. The improvement adaptive learning approach (IALA) is applied to all of the 192 problems. The proposed IALA method for NW-FSSP is compared with Aldowaisan and Allahverdi's ${ }^{2}$ results by using Genetic heuristic. The results of computational experiments on randomly generated NW-FSSPs are show that the proposed adaptive learning approach performs quite well.
\end{abstract}

Keywords: No-wait flowshop; Adaptive learning approach; Genetic algorithm; Makespan

\section{Introduction}

In the permutation flowshop, $n$ different jobs have to be processed on $m$ machines. Each job has one operation on each machine and all jobs have the same ordering sequencing on each machine. At any time, each machine can process at most one job. Preemption is not allowed ${ }^{3}$. An important kind of flowshop scheduling problem is characterized by a no-wait environment ${ }^{4}$. In a NW-
FSSP, jobs are not allowed to wait between two successive machines. This implies that the starting time of a job at the first machine has to be delayed to ensure that the job can go through the flow shop without having to wait for any machine ${ }^{5}$. The NW-FSSP has important applications in many industries. Examples include the metal, plastic and chemical industries, food and pharmaceutical industries. Additional applications can be found in advanced manufacturing environments,

* Corresponding Author: Orhan Engin

E-mail: orhanengin@yahoo.com, Tel: +90-332-2232039, Fax:+90-332-2410635 
such as just-in-time and flexible manufacturing systems $^{6}$.

The NW-FSSP has attracted the attention of many researchers. Reddi and Ramamoorthy ${ }^{7}$ and Wismer $^{8}$ were the first to address the NW-FSSP with the single criterion of makespan. Bonney and Gundry ${ }^{9}$ and King and Spachis $^{10}$ have later developed heuristics. Gangadharan and Rajendran ${ }^{11}$ and Rajendran ${ }^{12}$ have developed additional heuristics, and showed that their heuristics outperform those of Bonney and Gundry ${ }^{9}$ and King and Spachis ${ }^{10}$. Bertoliss ${ }^{13}$ presented a heuristic algorithm for NW-FSSPs to minimize the sum of the total flowtimes and at the same time minimizes the average processing time. Aldowaisan ${ }^{14}$ developed a new heuristic method for two-machine no-wait flowshop problem with separate setup times from processing times and sequence independent. Aldowaisan and Allahverdi ${ }^{2}$ presented two new heuristics that are based on simulated annealing and genetic algorithm techniques for no-wait flowshops to minimize makespan by incorporating a modified Nawaz-Enscore-Ham (NEH) heuristic (see Nawaz et al. ${ }^{15}$ ), which were shown to outperform those of Gangadharan and Rajendran ${ }^{11}$ and Rajendran ${ }^{12}$. Later, Aldowaisan and Allahverdi ${ }^{6}$ presented several new heuristics for the $m$-machine nowait flowshop with total completion time as the criterion. Shyu et al. ${ }^{16}$ developed an ant colony optimization for NW-FSSP to minimize the total completion time. Wang and $\mathrm{Cheng}^{4}$ proposed a heuristic approach for two-machine no-wait flowshop scheduling with due dates and class setups. Lin et al. ${ }^{17}$ developed a heuristic genetic algorithm for NW-FSSP. The authors compared the performance of the developed heuristic genetic algorithm with the heuristic proposal by Aldowaisan and Allahverdi ${ }^{6}$ Rahimi-Vahed ${ }^{18}$ considered a multi-objective scatter search for a bicriteria NW-FSSP in which weighted mean completion time and weighted mean tardiness are to be minimized simultaneously. Pan et al. ${ }^{19}$ proposed an iterated greedy algorithm for NW-FSSP with the objective to minimize the makespan and also Pan et al. ${ }^{20}$ presented a discrete particle swarm optimization for the NW-FSSP with both makespan and total flowtime criteria. TavakkoliMoghaddam et al. ${ }^{21}$ proposed an immune algorithm for a multi-objective NW-FSSP by minimizing the weighted mean completion time and weighted mean tardiness simultaneously. Laha and Chakraborty ${ }^{22}$ presented a new constructive heuristic, based on the principle of job insertion, for minimizing makespan in NW-FSSPs. Qian et al. ${ }^{23}$ proposed a memetic algorithm based on differential evolution for the multi-objective NW-FSSPs. Later, Qian et al. ${ }^{24}$ proposed an effective hybrid differential evolution for the NW-FSSP with the makespan criterion.
The aim of this paper is to suggest an adaptive learning approach for NW-FSSP with makespan criteria. The performance of the proposed approach is tested on a set of 192 instances. The comparison is made with the Aldowaisan and Allahverdi's ${ }^{2}$ results by using Genetic heuristic. The rest of this article is organized as follows. Section 2 provides adaptive learning approach. Genetic heuristic approach is given in Section 3. Section 4 presents computational results. Finally, the conclusions about adaptive learning approach are presented in Section 5.

\section{Adaptive Learning Approach}

Adaptive learning approach is similar to neural-network training. Numerous neural network models have been developed for solving optimization problem in the past two decades. The most popular seminal network model for optimization problem is Hopfield and Tank ${ }^{25}$ model. During the past decade, numerous neural network models have been developed for solving the scheduling problems. Park et al. ${ }^{26}$ proposed an approach for solving a parallel-machine scheduling problem applying a known heuristic rule combining it with a neural network technique. Lee and Shaw ${ }^{27}$ presented a neural-net approach for real time flow-shop sequencing. They apply the neural-net approach to construct a sequence for a set of jobs that arrive in different job combinations over time. Fonseca and Navaresse ${ }^{28}$ proposed an artificial neural network for the traditional job-shop simulation. Solimanpur et al. $^{29}$ proposed a neural networks-based tabu search method, for the flow shop scheduling problem. Agarwal et al. ${ }^{1}$ propose an improvement-heuristic approach for the general flowshop problem based on the idea of adaptive learning. Also, Agarwal et al. ${ }^{30}$ proposed new heuristics along with an augmented-neural-network formulation for solving the makespan minimization task-scheduling problem for the non-identical machine environment. Akyol and Bayhan ${ }^{31}$ presented a review on evolution of production scheduling with neural networks.

In this study, Agarwal et al.'s ${ }^{1}$ adaptive learning approach (ALA) is improvement for no-wait flowshop scheduling problems. The general idea is that Agarwal et al. ${ }^{1}$ define a weight factor associated with each operation. They use weighted processing times instead of regular processing times and also weights are modified using a certain strategy.

\section{Genetic Heuristic Approach}

Genetic algorithm (GA) is a stochastic optimization technique for solving the scheduling problem. GAs are based on natural selection and genetics. They were first developed by John Holland ${ }^{32}$ in 1975. GAs use a 
collection of solutions called population. Each individual in the population is called a chromosome and a chromosome represents a solution to the problem. The chromosomes can be produced through successive iterations, called generations also the chromosomes are evaluated using the value of fitness function during each generation $^{33}$.

In this study the improvement learning approach for NW-FSSP is compared with Aldowaisan and Allahverdi's ${ }^{2}$ results by using Genetic heuristic (GEN2). A brief outline of the Aldowaisan and Allahverdi's ${ }^{2}$ Genetic heuristic is given below, where the notation $P(t)$ denotes the population at the $t$ th generation, $s_{i}(t)$ represents the $i$ th job sequence in $P(t)$ and $f\left(s_{i}(t)\right)$ is the fitness value of $s_{i}(t)$.

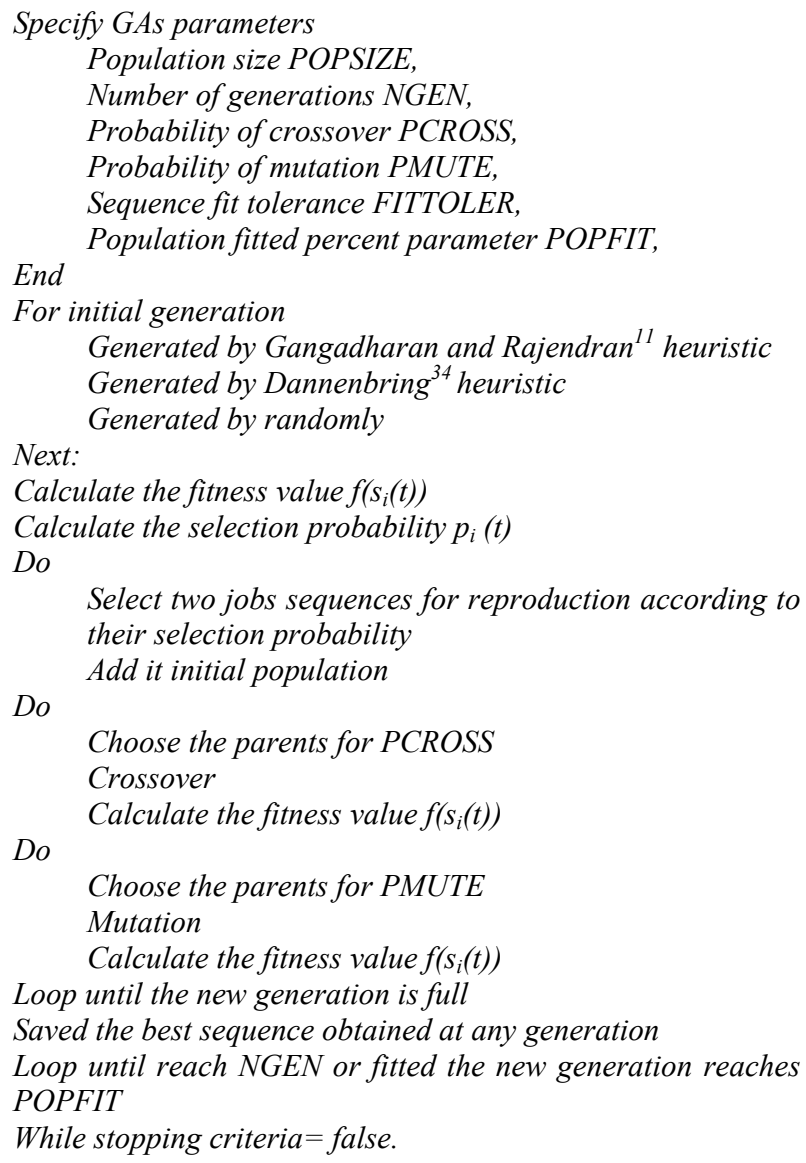

Calculate the fitness value $f\left(s_{i}(t)\right)$

Calculate the selection probability $p_{i}(t)$

Do

Select two jobs sequences for reproduction according to their selection probability

Do Add it initial population

Choose the parents for PCROSS

Crossover Do

Calculate the fitness value $f\left(s_{i}(t)\right)$

Choose the parents for PMUTE

Mutation

Calculate the fitness value $f\left(s_{i}(t)\right)$

Loop until the new generation is full

Saved the best sequence obtained at any generation

Loop until reach NGEN or fitted the new generation reaches POPFIT

While stopping criteria $=$ false.

Table 1 lists the parameters used in Aldowaisan and Allahverdi's ${ }^{2}$ best Genetic heuristic(GEN-2).
Table 1. Best GEN-2² Parameters List

\begin{tabular}{ll}
\hline Parameter & Value \\
\hline Population size & 95 \\
Number of generations & 60 \\
Fitted tolerance & $1 \mathrm{E}-10$ \\
Population fitted percent & 60 \\
Probability of crossover & 0.725 \\
Probability of mutation & 0.009 \\
\hline
\end{tabular}

The Aldowaisan and Allahverdi's ${ }^{2}$ best GEN-2 heuristic is summarized in Table 2.

Table 2. Best GEN-2 heuristic method ${ }^{2}$

\begin{tabular}{|c|l|l|}
\hline Heuristic & Initial solution & $\begin{array}{l}\text { New solution } \\
\text { generation }\end{array}$ \\
\hline & $\begin{array}{l}\text { Gangadharan and } \\
\text { Rajendran }^{11}\end{array}$ & $\begin{array}{l}\text { Roulette wheel } \\
\text { selection, }\end{array}$ \\
GEN-2 & $\begin{array}{l}\text { Dannenbring } \\
\text { Campbell Dudek } \\
\text { Smith }\end{array}$ & $\begin{array}{l}\text { Partially } \\
\text { Mapped } \\
\text { Crossover-PMX }\end{array}$ \\
Random & Swapping \\
\hline
\end{tabular}

\section{Computational Results}

In this study, to evaluate the performance of the improvement adaptive learning approach for no-wait flowshop scheduling, 192 problems are generated. The processing times on each machine were randomly generated from a discrete uniform distribution from the different intervals for, $a$ type problems $[1,10], b$ type problems $[1,50]$ and $c$ type problems $[1,100]$ which is commonly used in the literature e.g ${ }^{16,2,12}$. Also the values of setup time, s, on each job were randomly generated from a discrete uniform distribution the interval $[1,10]$ which is commonly used in the literature.

The number of jobs $(n)$ considered is $8,10,12,50,100$, $150,200,250$, and the number of machines $(m)$ is 2,3 , $5,8,10,15,20$, and 25 which is commonly used in the literature. The improvement Agarwal et al.'s ${ }^{1}$ IALA for no-wait flowshop scheduling problems notation and algorithm is given below.

$\begin{array}{ll}\text { Notation } & \\ n & \text { Set of } n \text { jobs } \\ \mathrm{m} & \text { Set of } m \text { machines } \\ O_{i j} & \text { Operation of } i \text { th job on } j \text { th machine } \\ T_{i j} & \text { Processing time of operation } O_{i j} \\ W_{i j} & \text { Weighted associated time of operation } O_{i j} \\ W T_{i j} & \text { Weighted processing time of operation } O_{i j} \\ k & \text { Iteration number }\end{array}$




$\begin{array}{ll}K_{\max } & \text { Max number of iteration } \\ M S_{k} & \text { Makespan in the } k \text { th iteration } \\ S_{i} & \text { Set up time of } i \text { th on each machine } \\ R F & \text { Reinforcement factor } \\ T I N I & \text { Tolerate iterations with no improvement } \\ \alpha & \text { Learning rate } \\ B M S & \text { Best makespan } \\ B W_{i j} & \text { Best weights } \\ R N D & \text { Random number }\end{array}$

The proposed IALA method can be summarized as follows;

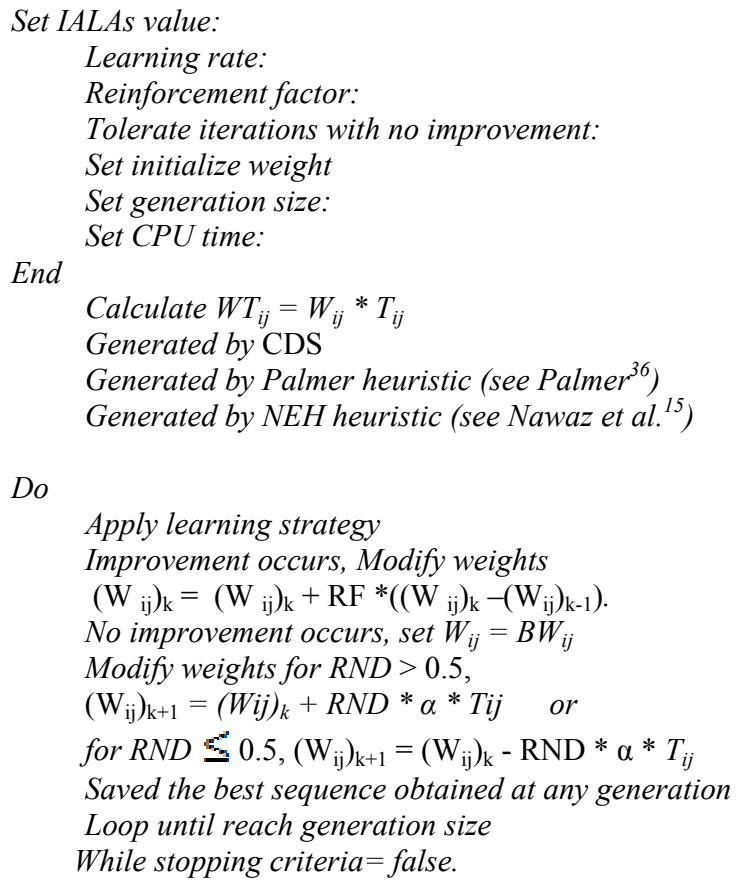

The initialize weight is set 1 for all jobs. In the IALA method, three learning parameters, namely, the learning rate $(\alpha)$, the reinforcement factor $(R F)$ and the tolerate iterations with no improvement (TINI) are used. These parameters were explained by Agarwal et al ${ }^{1}$.

It is well known that the IALAs' efficiency depends to a high degree upon to the selection of the learning parameters. The determination of the suitable settings for the learning parameters of IALA is very difficult task. In general, there are a few control mechanisms for these learning parameters, and the full factorial design of experiments is used in this study for efficiency of IALA to solve the NW-FSSP. The application involved three learning parameters, each having possible different values.

These parameters are given as follows

\section{Reinforcement factor: 1, 2, 3, 4, 5, 6, 7, 8, 9, 10}

Tolerate iterations with no improvement: 10, 20, 30, 40, and 50

The number of replicates is 25 . In total, 192 problems are classified into 8 groups depending on the number of machines and an instance problem is taken from each group. The parameter optimization is implemented and the best parameter set is determined for the instance. The best parameter set determined for an instance is generalized and used for the other problems of the same group. Therefore, the parameter optimization is implemented for 64 instances. Consequently, 10 learning rate levels, 10 reinforcement factor levels and 5 tolerate iterations with no improvement levels are implemented among the 64 problems with the 25 replicated.

A total number of $10 * 10 * 5 * 25=12,500$ runs are made among the 64 problems. Table 3 shows the parameter setting in each of the replicated runs.

There are 192 instances and $n x m-a, n x m-b$ and $n x m-c$ problems defined as having $n$-jobs $(n=8,10,12,50$, $100,150,200,250), m$-machine $(m=2,3,5,8,10,15$, $20,25)$ and $a, b$ and $c$ type problems. The processing times of $a$ type problems are $[1,10]$ interval, $b$ type problems $[1,50]$ interval and $c$ type problems $[1,100]$ interval.

Our computational study aims to seek a good heuristic solution for the NW-FSSPs. The results are compared with the Aldowaisan and Allahverdi's (2003) results by using Genetic heuristic (GEN-2). The algorithm is implemented in Borland Delphi and is executed with a PC with a Pentium IV with $3.0 \mathrm{GHz}$ processor and $1 \mathrm{~GB}$ memory.

The results obtained with the IALA and GEN-2 and the comparisons of these two heuristic are presented in Table 4. Percentage Deviation (PD) is defined as follows.

For the $I A L A$

$P D=\left(\left(\operatorname{IALA}\left(C_{\max }\right)-\operatorname{Best}\left(C_{\max }\right)\right) / \operatorname{Best}\left(C_{\max }\right)\right) * 100$

For the $G E N-2$

$P D=\left(\left(G E N-2\left(C_{\max }\right)-\operatorname{Best}\left(C_{\max }\right)\right) / \operatorname{Best}\left(C_{\max }\right)\right) * 100$

Learning rate: $0.01,0.02,0.03,0.04,0.05,0.06,0.07$, $0.08,0.09,0.100$ 
Table 3. Best parameter sets

\begin{tabular}{|c|c|c|c|c|}
\hline $\begin{array}{c}\text { Machine } \\
\text { (m) }\end{array}$ & $\begin{array}{l}\text { Job } \\
\text { (n) }\end{array}$ & $\boldsymbol{R} F$ & $T I N I$ & $\alpha$ \\
\hline \multirow{3}{*}{2} & $\begin{array}{c}8 \\
10 \\
12 \\
\end{array}$ & 3 & 30 & 0.02 \\
\hline & $\begin{array}{c}50 \\
100 \\
150\end{array}$ & 7 & 50 & 0.05 \\
\hline & $\begin{array}{l}200 \\
250\end{array}$ & 5 & 40 & 0.06 \\
\hline \multirow{3}{*}{3} & $\begin{array}{c}8 \\
10 \\
12\end{array}$ & 2 & 20 & 0.07 \\
\hline & $\begin{array}{c}50 \\
100 \\
150 \\
\end{array}$ & 5 & 40 & 0.04 \\
\hline & $\begin{array}{l}200 \\
250 \\
\end{array}$ & 5 & 40 & 0.06 \\
\hline \multirow{3}{*}{5} & $\begin{array}{c}8 \\
10 \\
12\end{array}$ & 2 & 10 & 0.02 \\
\hline & $\begin{array}{c}50 \\
100 \\
150\end{array}$ & 7 & 50 & 0.06 \\
\hline & $\begin{array}{l}200 \\
250\end{array}$ & 8 & 50 & 0.07 \\
\hline \multirow{3}{*}{8} & $\begin{array}{c}8 \\
10 \\
12\end{array}$ & 2 & 50 & 0.02 \\
\hline & $\begin{array}{c}50 \\
100 \\
150 \\
\end{array}$ & 4 & 30 & 0.08 \\
\hline & $\begin{array}{l}200 \\
250\end{array}$ & 10 & 40 & 0.09 \\
\hline
\end{tabular}

\begin{tabular}{|c|c|c|c|c|}
\hline $\begin{array}{c}\text { Machine } \\
\text { (m) }\end{array}$ & $\begin{array}{c}J o b \\
(n)\end{array}$ & $\boldsymbol{R F}$ & TINI & $\alpha$ \\
\hline \multirow{8}{*}{10} & 8 & \multirow{3}{*}{4} & \multirow{3}{*}{40} & \multirow{3}{*}{0.03} \\
\hline & 10 & & & \\
\hline & 12 & & & \\
\hline & 50 & \multirow{3}{*}{4} & \multirow{3}{*}{20} & \multirow{3}{*}{0.01} \\
\hline & 100 & & & \\
\hline & 150 & & & \\
\hline & 200 & \multirow{2}{*}{6} & \multirow{2}{*}{40} & \multirow{2}{*}{0.06} \\
\hline & 250 & & & \\
\hline \multirow{8}{*}{15} & 8 & \multirow{3}{*}{3} & \multirow{3}{*}{40} & \multirow{3}{*}{0.07} \\
\hline & 10 & & & \\
\hline & 12 & & & \\
\hline & 50 & \multirow{3}{*}{6} & \multirow{3}{*}{30} & \multirow{3}{*}{0.08} \\
\hline & 100 & & & \\
\hline & 150 & & & \\
\hline & 200 & \multirow{2}{*}{9} & \multirow{2}{*}{40} & \multirow{2}{*}{0.06} \\
\hline & 250 & & & \\
\hline \multirow{8}{*}{20} & 8 & \multirow{3}{*}{4} & \multirow{3}{*}{30} & \multirow{3}{*}{0.08} \\
\hline & 10 & & & \\
\hline & 12 & & & \\
\hline & 50 & \multirow{3}{*}{6} & \multirow{3}{*}{20} & \multirow{3}{*}{0.06} \\
\hline & 100 & & & \\
\hline & 150 & & & \\
\hline & 200 & \multirow{2}{*}{8} & \multirow{2}{*}{30} & \multirow{2}{*}{0.01} \\
\hline & 250 & & & \\
\hline & 8 & & & \\
\hline & 10 & 6 & 50 & 0.02 \\
\hline & 12 & & & \\
\hline & 50 & & & \\
\hline 25 & 100 & 5 & 20 & 0.07 \\
\hline & 150 & & & \\
\hline & 200 & 9 & 20 & 0.05 \\
\hline & 250 & 9 & 20 & \\
\hline
\end{tabular}


Table 4. Heuristics comparison

\begin{tabular}{|c|c|c|c|c|c|c|c|c|}
\hline \multirow{2}{*}{ Problem } & \multicolumn{2}{|c|}{ PD } & \multirow{2}{*}{ Problem } & \multicolumn{2}{|c|}{ PD } & \multirow{2}{*}{ Problem } & \multicolumn{2}{|c|}{ PD } \\
\hline & GEN-2 & IALA & & GEN-2 & IALA & & GEN-2 & IALA \\
\hline $8 \times 2-a$ & 0.000 & 0.000 & $8 \times 3-a$ & 0.000 & 0.000 & $8 \times 5-a$ & 0.794 & 0.000 \\
\hline $8 \times 2-b$ & 0.000 & 0.000 & $8 \times 3-b$ & 0.000 & 4.828 & $8 \times 5-b$ & 0.000 & 0.505 \\
\hline $8 \times 2-c$ & 0.000 & 0.645 & $8 \times 3-c$ & 0.534 & 0.000 & $8 \times 5-c$ & 1.075 & 0.000 \\
\hline $10 \times 2-a$ & 0.000 & 0.000 & $10 \times 3-a$ & 0.000 & 0.840 & $10 \times 5-a$ & 1.429 & 0.000 \\
\hline $10 \times 2-b$ & 0.000 & 1.775 & $10 \times 3-b$ & 3.270 & 0.000 & $10 \times 5-b$ & 0.000 & 3.846 \\
\hline $10 \times 2-c$ & 1.541 & 0.000 & $10 \times 3-c$ & 0.727 & 0.000 & $10 \times 5-c$ & 0.885 & 0.000 \\
\hline $12 \times 2-a$ & 0.000 & 0.000 & $12 \times 3-a$ & 0.000 & 0.000 & $12 \times 5-a$ & 0.000 & 1.149 \\
\hline $12 \times 2-b$ & 0.000 & 0.000 & $12 \times 3-b$ & 1.790 & 0.000 & $12 \times 5-b$ & 1.034 & 0.000 \\
\hline $12 \times 2-c$ & 1.387 & 0.000 & $12 \times 3-c$ & 0.000 & 1.952 & $12 \times 5-c$ & 0.000 & 1.473 \\
\hline $50 \times 2-a$ & 1.054 & 0.000 & $50 \times 3-a$ & 0.000 & 1.124 & $50 \times 5-a$ & 0.419 & 0.000 \\
\hline $50 \times 2-b$ & 0.000 & 1.461 & $50 \times 3-b$ & 1.710 & 0.000 & $50 \times 5-b$ & 0.665 & 0.000 \\
\hline $50 \times 2-c$ & 1.158 & 0.000 & $50 \times 3-c$ & 0.292 & 0.000 & $50 \times 5-c$ & 0.000 & 3.213 \\
\hline $100 \times 2-a$ & 0.719 & 0.000 & $100 \times 3-a$ & 0.000 & 0.722 & $100 \times 5-a$ & 0.756 & 0.000 \\
\hline $100 \times 2-b$ & 0.000 & 0.167 & $100 \times 3-b$ & 0.547 & 0.000 & $100 \times 5-b$ & 0.538 & 0.000 \\
\hline $100 \times 2-c$ & 0.000 & 0.240 & $100 \times 2-c$ & 0.573 & 0.000 & $100 \times 5-c$ & 0.571 & 0.000 \\
\hline $150 \times 2-a$ & 0.475 & 0.000 & $150 \times 3-a$ & 0.154 & 0.000 & $150 \times 5-a$ & 0.000 & 0.275 \\
\hline $150 \times 2-b$ & 0.000 & 0.477 & $150 \times 3-b$ & 0.113 & 0.000 & $150 \times 5-b$ & 0.198 & 0.000 \\
\hline $150 \times 2-c$ & 0.102 & 0.000 & $150 \times 3-c$ & 0.371 & 0.000 & $150 \times 5-c$ & 1.031 & 0.000 \\
\hline $200 \times 2-a$ & 0.482 & 0.000 & $200 \times 3-a$ & 0.157 & 0.000 & $200 \times 5-a$ & 0.343 & 0.000 \\
\hline $200 \times 2-b$ & 0.000 & 0.473 & $200 \times 3-b$ & 0.000 & 0.368 & $200 \times 5-b$ & 0.159 & 0.000 \\
\hline $200 \times 2-c$ & 0.483 & 0.000 & $200 \times 3-c$ & 1.303 & 0.000 & $200 \times 5-c$ & 0.000 & 0.503 \\
\hline $250 \times 2-a$ & 0.000 & 0.821 & $250 \times 3-a$ & 0.000 & 0.124 & $250 \times 5-a$ & 0.273 & 0.000 \\
\hline $250 \times 2-b$ & 0.531 & 0.000 & $250 \times 3-b$ & 0.235 & 0.000 & $250 \times 5-b$ & 0.325 & 0.000 \\
\hline $250 \times 2-c$ & 0.035 & 0.000 & $250 \times 3-c$ & 0.766 & 0.000 & $250 \times 5-c$ & 0.332 & 0.000 \\
\hline $8 \times 8-a$ & 0.000 & 0.000 & $8 \times 10-a$ & 0.658 & 0.000 & $8 \times 15-a$ & 0.000 & 0.000 \\
\hline $8 \times 8-b$ & 0.000 & 0.000 & $8 \times 10-b$ & 0.000 & 0.000 & $8 \times 15-b$ & 0.000 & 0.000 \\
\hline $8 \times 8-c$ & 0.000 & 0.000 & $8 \times 10-c$ & 0.000 & 0.602 & $8 \times 15-c$ & 0.000 & 0.000 \\
\hline $10 \times 8-a$ & 0.000 & 0.000 & $10 \times 10-a$ & 0.000 & 1.099 & $10 \times 15-a$ & 0.905 & 0.000 \\
\hline $10 \times 8-b$ & 0.000 & 0.809 & $10 \times 10-b$ & 0.000 & 3.823 & $10 \times 15-b$ & 0.910 & 0.000 \\
\hline $10 \times 8-c$ & 0.000 & 0.000 & $10 \times 10-c$ & 5.049 & 0.000 & $10 \times 15-c$ & 0.000 & 1.426 \\
\hline $12 \times 8-a$ & 0.995 & 0.000 & $12 \times 10-a$ & 0.943 & 0.000 & $12 \times 15-a$ & 0.760 & 0.000 \\
\hline $12 \times 8-b$ & 1.108 & 0.000 & $12 \times 10-b$ & 0.000 & 2.522 & $12 \times 15-b$ & 0.484 & 0.000 \\
\hline $12 \times 8-c$ & 4.808 & 0.000 & $12 \times 10-c$ & 2.291 & 0.000 & $12 \times 15-c$ & 1.403 & 0.000 \\
\hline $50 \times 8-a$ & 2.261 & 0.000 & $50 \times 10-a$ & 0.231 & 0.000 & $50 \times 15-a$ & 0.000 & 1.117 \\
\hline $50 \times 8-b$ & 1.364 & 0.000 & $50 \times 10-b$ & 0.500 & 0.000 & $50 \times 15-b$ & 0.158 & 0.000 \\
\hline $50 \times 8-c$ & 0.089 & 0.000 & $50 \times 10-c$ & 0.276 & 0.000 & $50 \times 15-c$ & 0.000 & 0.920 \\
\hline $100 \times 8-a$ & 1.094 & 0.000 & $100 \times 10-a$ & 0.228 & 0.000 & $100 \times 15-a$ & 0.866 & 0.000 \\
\hline $100 \times 8-b$ & 0.000 & 0.446 & $100 \times 10-b$ & 0.000 & 0.339 & $100 \times 15-b$ & 0.000 & 0.761 \\
\hline $100 \times 8-c$ & 0.575 & 0.000 & $100 \times 10-c$ & 0.000 & 0.080 & $100 \times 15-c$ & 1.401 & 0.000 \\
\hline $150 \times 8-a$ & 0.493 & 0.000 & $150 \times 10-a$ & 0.190 & 0.000 & $150 \times 15-a$ & 0.136 & 0.000 \\
\hline $150 \times 8-b$ & 0.023 & 0.000 & $150 \times 10-b$ & 0.898 & 0.000 & $150 \times 15-b$ & 0.992 & 0.000 \\
\hline $150 \times 8-c$ & 0.000 & 0.665 & $150 \times 10-c$ & 0.890 & 0.000 & $150 \times 15-c$ & 0.773 & 0.000 \\
\hline $200 \times 8-a$ & 0.152 & 0.000 & $200 \times 10-a$ & 0.000 & 0.086 & $200 \times 15-a$ & 0.903 & 0.000 \\
\hline $200 \times 8-b$ & 0.271 & 0.000 & $200 \times 10-b$ & 1.159 & 0.000 & $200 \times 15-b$ & 0.020 & 0.000 \\
\hline $200 \times 8-c$ & 0.813 & 0.000 & $200 \times 10-c$ & 0.000 & 0.036 & $200 \times 15-c$ & 0.000 & 0.302 \\
\hline $250 \times 8-a$ & 0.000 & 0.340 & $250 \times 10-a$ & 0.135 & 0.000 & $250 \times 15-a$ & 0.162 & 0.000 \\
\hline $250 \times 8-b$ & 0.000 & 0.080 & $250 \times 10-b$ & 0.979 & 0.000 & $250 \times 15-b$ & 1.022 & 0.000 \\
\hline $250 \times 8-c$ & 0.069 & 0.000 & $250 \times 10-c$ & 0.309 & 0.000 & $250 \times 15-c$ & 0.000 & 0.035 \\
\hline
\end{tabular}


Table 4. (Continued)

\begin{tabular}{|c|c|c|}
\hline \multirow[b]{2}{*}{ Problem } & \multicolumn{2}{|c|}{ PD } \\
\hline & $\begin{array}{c}\text { GEN- } \\
2\end{array}$ & IALA \\
\hline $8 \times 20-a$ & 0.463 & 0.000 \\
\hline $8 \times 20-b$ & 0.000 & 0.000 \\
\hline $8 \times 20-c$ & 0.000 & 0.000 \\
\hline $10 \times 20-a$ & 0.763 & 0.000 \\
\hline $10 \times 20-b$ & 0.833 & 0.000 \\
\hline $10 \times 20-c$ & 0.858 & 0.000 \\
\hline $12 \times 20-a$ & 0.000 & 3.136 \\
\hline $12 \times 20-b$ & 0.000 & 0.000 \\
\hline $12 \times 20-c$ & 2.285 & 0.000 \\
\hline $50 \times 20-a$ & 0.189 & 0.000 \\
\hline $50 \times 20-b$ & 0.568 & 0.000 \\
\hline $50 \times 20-c$ & 0.000 & 0.096 \\
\hline $100 \times 20-a$ & 0.235 & 0.000 \\
\hline $100 \times 20-b$ & 0.523 & 0.000 \\
\hline $100 \times 20-c$ & 1.630 & 0.000 \\
\hline $150 \times 20-\mathrm{a}$ & 0.063 & 0.000 \\
\hline $150 \times 20-b$ & 0.000 & 0.110 \\
\hline $150 \times 20-c$ & 0.498 & 0.000 \\
\hline $200 \times 20-a$ & 0.000 & 0.379 \\
\hline $200 \times 20-b$ & 0.365 & 0.000 \\
\hline $200 \times 20-c$ & 0.000 & 0.450 \\
\hline $250 \times 20-a$ & 0.261 & 0.000 \\
\hline $250 \times 20-b$ & 0.442 & 0.000 \\
\hline $250 \times 20-c$ & 0.434 & 0.000 \\
\hline
\end{tabular}

\begin{tabular}{lcc}
\hline Problem & \multicolumn{2}{c}{ GEN- } \\
& $\mathbf{2}$ & IALA \\
\hline $8 \times 25-\mathrm{a}$ & 0.000 & 0.000 \\
$8 \times 25-\mathrm{b}$ & 0.842 & 0.000 \\
$8 \times 25-\mathrm{c}$ & 2.487 & 0.000 \\
$10 \times 25-\mathrm{a}$ & 0.352 & 0.000 \\
$10 \times 25-\mathrm{b}$ & 0.160 & 0.000 \\
$10 \times 25-\mathrm{c}$ & 1.632 & 0.000 \\
$12 \times 25-\mathrm{a}$ & 0.597 & 0.000 \\
$12 \times 25-\mathrm{b}$ & 0.349 & 0.000 \\
$12 \times 25-\mathrm{c}$ & 0.000 & 0.177 \\
$50 \times 25-\mathrm{a}$ & 0.086 & 0.000 \\
$50 \times 25-\mathrm{b}$ & 0.534 & 0.000 \\
$50 \times 25-\mathrm{c}$ & 0.729 & 0.000 \\
$100 \times 25-\mathrm{a}$ & 0.000 & 0.214 \\
$100 \times 25-\mathrm{b}$ & 0.158 & 0.000 \\
$100 \times 25-\mathrm{c}$ & 0.560 & 0.000 \\
$150 \times 25-\mathrm{a}$ & 0.116 & 0.000 \\
$150 \times 25-\mathrm{b}$ & 0.924 & 0.000 \\
$150 \times 25-\mathrm{c}$ & 0.000 & 0.673 \\
& & \\
$200 \times 25-\mathrm{a}$ & 0.131 & 0.000 \\
$200 \times 25-\mathrm{b}$ & 1.598 & 0.000 \\
$200 \times 25-\mathrm{c}$ & 0.055 & 0.000 \\
$250 \times 25-\mathrm{a}$ & 0.295 & 0.000 \\
$250 \times 25-\mathrm{b}$ & 0.000 & 0.809 \\
$250 \times 25-\mathrm{c}$ & 0.616 & 0.000 \\
\hline & &
\end{tabular}

As it seen in Table 4, for 2-machines problems the proposed IALA found best- $\mathrm{C}_{\max }$ values for 16 problems over 24 while GEN-2 found only 13 best- $\mathrm{C}_{\max }$ values. When all problems are considered, the proposed IALA found best- $\mathrm{C}_{\max }$ values for 142 problems over 192 while GEN-2 found only 70 best- $C_{\max }$ values. These results are summarized in Table 5.

Table 5. The performance of IALA and GEN-2

\begin{tabular}{ccc}
\hline \multirow{2}{*}{$\begin{array}{c}\text { Problems } \\
\text { For- machines }\end{array}$} & \multicolumn{2}{c}{ Best-C $_{\max }$} \\
\cline { 2 - 3 } & GEN-2 & $\begin{array}{c}\text { Proposed } \\
\text { IALA }\end{array}$ \\
\hline 2 & 13 & 16 \\
3 & 9 & 17 \\
5 & 7 & 17 \\
8 & 10 & 19 \\
10 & 9 & 16 \\
15 & 9 & 18 \\
20 & 8 & 19 \\
25 & 5 & 20 \\
\hline Total & $\mathbf{7 0}$ & $\mathbf{1 4 2}$ \\
\hline
\end{tabular}

Average Percentage Deviation (APD) of proposed IALA is also compared with GEN-2. The APD of proposed IALA and GEN-2 are defined as follows;

$$
A P D=\frac{\sum_{L=1}^{I} P D(L)}{I}
$$

The results are presented in Table 6 .

Table 6 The APD computational results of IALA and GEN-2

\begin{tabular}{ccc}
\hline \multirow{2}{*}{$\begin{array}{c}\text { Problems } \\
\text { For machines }\end{array}$} & GEN-2 & $\begin{array}{c}\text { Proposed } \\
\text { IALA }\end{array}$ \\
\cline { 2 - 3 } & & 0.252 \\
2 & 0.332 & 0.415 \\
3 & 0.523 & 0.457 \\
5 & 0.451 & 0.097 \\
10 & 0.588 & 0.358 \\
15 & 0.614 & 0.237 \\
20 & 0.454 & 0.174 \\
25 & 0.434 & 0.078 \\
\hline Total & 0.509 & $\mathbf{0 . 2 5 8 5}$ \\
Average & $\mathbf{0 . 4 8 8 1}$ & \\
\hline
\end{tabular}

As it seen in Table 6 for all problems average APD for proposed IALA and GEN-2 is 0.2585 and 0.4881 respectively. Proposed IALA has found the smaller average APD than GEN-2.

The CPU times of proposed IALA and GEN-2 are compared in Table 7.

Table 7 The maximum CPU times of the IALA and GEN-2

\begin{tabular}{llc}
\hline Methods & $\begin{array}{l}\text { Configuration } \\
\text { of the computer }\end{array}$ & $\begin{array}{c}\text { Maximum } \\
\text { CPU times } \\
\text { (sec) }\end{array}$ \\
\hline $\begin{array}{l}\text { Proposed } \\
\text { IALA }\end{array}$ & $\begin{array}{l}\text { PC Pentium IV-3.0 } \\
\text { GHz- 1 GB } \\
\text { memory }\end{array}$ & 110 \\
\hline GEN-2 & $\begin{array}{l}\text { PC Pentium IV-3.0 } \\
\text { GHz- 1 GB } \\
\text { memory }\end{array}$ & 156 \\
\hline
\end{tabular}


The computational results indicated that the proposed IALA method is effective on average in terms of a reduced makespan for the NW-FSSP.

\section{Conclusions}

In this paper, an adaptive learning approach is suggested for NW-FSSP with the makespan criteria. The considered problem is a NP-Hard problem. Most of the studies to solve that problem are approximate methods rather than an exact method. The test problem are generated according to the literature e.g., Shyu et al. ${ }^{16}$, Aldowaisan and Allahverdi ${ }^{2}$, Gangadharan and Rajendran $^{11}$ and Rajendran ${ }^{12}$. The percentage deviations from Aldowaisan and Allahverdi's ${ }^{2}$ results by using Genetic heuristic (GEN-2) are calculated. The better solutions are resulted with the suggested adaptive learning approach. The proposed adaptive learning approach is a good problem solving technique for NWFSSP with the makespan criteria on average.

For further research, some changes may be done in the learning strategy or the algorithm may be hibritted with another method to improve solution quality.

\section{References}

1. A. Agarwal, S. Colak, E. Eryarsoy, Improvement heuristic for the flow-shop scheduling problem: An adaptive-learning approach, European Journal of Operational Research 169 (2006) 801-815.

2. T. Aldowaisan, and A. Allahverdi, New heuristics for nowait flowshops to minimize makespan, Computers \& Operations Research 30 (8) (2003) 1219-1231.

3. D.E. Akyol, Application of neural networks to heuristic scheduling algorithms, Computers \& Industrial Engineering 46 (2004) 679- 696.

4. X. Wang, and T.C.E. Cheng, A heuristic approach for two-machine no-wait flowshop scheduling with due dates and class setups, Computers \& Operations Research 33 (2006) 1326-1344.

5. M.L. Pinedo, Scheduling, Theory, Algorithm, and Systems, Third Edition (Springer, New York, 2008).

6. T. Aldowaisan, and A. Allahverdi, New heuristics for $m$-machine no-wait flowshop to minimize total completion time, Omega 32 (5) (2004) 345-352.

7. S.S. Reddi, C.V. Ramamoorthy, On the flowshop sequencing problem with no-wait in process, Operational Research Quarterly, 23 (3) (1972) 323-331.

8. D.A. Wismer, Solution of the flowshop scheduling problem with no intermediate queues, Operations Research, 20 (3) (1972) 689-697.

9. M.C. Bonney, and S.W. Gundry, Solutions to the constrained flowshop sequencing problem, Operational Research Quarterly, 27 (4) (1976) 869-883.
10. J.R. King, and A.S. Spachis, Heuristics for flowshop scheduling. International Journal of Production Research, 18 (3) (1980) 345-357.

11. R. Gangadharan, C. Rajendran, Heuristic algorithms for scheduling in the no-wait fowshop, International Journal of Production Economics 32 (3) (1993) 285- 290.

12. C. Rajendran, A no-wait flowshop scheduling heuristic to minimize makespan, Journal of the Operational Research Society, 45 (4) (1994) 472-478.

13. E. Bertolissi, Heuristic algorithm for scheduling in the no-wait flow-shop, Journal of Materials Processing Technology, 107 (2000) 459- 465.

14. T. Aldowaisan, A new heuristic and dominance relations for no-wait flowshops with setups, Computers \& Operations Research 28 (2001) 563- 584.

15. M. Nawaz, E. E. Enscore, and I. Ham, A heuristic algorithm for the m-machine, $n$-job flow-shop sequencing problem, Omega, 11 (1983) 91-95.

16. S.J. Shyu, B.M.T. Lin, P.Y. Yin, Application of ant colony optimization for no-wait flowshop scheduling problem to minimize the total completion time, Computers \& Industrial Engineering 47 (2004) 181193.

17. C. J. Lin, G. Dun-wei, M. Xiao-ping, A heuristic genetic algorithm for the no wait flowshop scheduling problem, Journal of China University of Mining and Technology, 17 (4) (2007) 582- 586.

18. A.R. Rahimi-Vahed, B. Javadi, M. Rabbani, R. Tavakkoli-Moghaddama, A multi-objective scatter search for a bi-criteria no-wait flow shop scheduling problem, Engineering Optimization, 40 (4) (2008) 331-346.

19. Q. K. Pan, L. Wang, B, H. Zhao, An improved iterated greedy algorithm for the no-wait flow shop scheduling problem with makespan criterion, Int $J A d v$ Manuf Technol, 38 (2008) 778-786.

20. Q. K. Pan, M. F. Tasgetiren, Y. C. Liang, A discrete particle swarm optimization algorithm for the no wait flowshop scheduling problem, Computers and Operations Research, 35 (9) (2008) 2807- 2839.

21. R. Tavakkoli-Moghaddam, A. R. Rahimi-Vahed, A.H. Mirzae, Solving a multi-objective no-wait flow shop scheduling problem with an immune algorithm, Int $J A d v$ Manuf Technol, 36 (2008) 969-981.

22. D. Laha, U.K. Chakraborty, A constructive heuristic for minimizing makespan in no-wait flow shop scheduling, Int J Adv Manuf Technol 41 (2009) 97-109.

23. B. Qian, L. Wang, D. X., Huang, X. Wang, Multiobjective no-wait flow-shop scheduling with a memetic algorithm based on differential evolution, Soft Comput, 13 (2009) 847-869

24. B. Qian, L. Wang, R. Hu, D.X. Huang, X. Wang, A DE-based approach to no-wait flow-shop scheduling, Computers \& Industrial Engineering, 57 (2009) $787-$ 805.

25. J. J. Hopfield, D.W. Tank, Neural computation of decisions in optimization problems, Biological Cybernetics 52 (3) (1985) 141- 152. 
26. Y. Park, S. Kim, Y. H. Lee, Scheduling jobs on parallel machines applying neural network and heuristic rules, Computers \& Industrial Engineering, 38 (2000) 189202.

27. I. Lee, M. J. Shaw, A neural-net approach to real time flow-shop sequencing, Computers \& Industrial Engineering, 38 (2000) 125- 147.

28. D. J. Fonseca, and D. Navaresse, Artificial neural networks for job shop simulation, Advanced Engineering Informatics 16 (2002) 241-246.

29. M. Solimanpur, P. Vrat, R. Shankar, A neuro-tabu search heuristic for the flow shop scheduling problem, Computers \& Operations Research 31 (2004) 21512164.

30. A. Agarwal, S. Colak, V. S. Jacob, H. Pirkul, Heuristics and augmented neural networks for task scheduling with non-identical machines, European Journal of Operational Research, 175 (2006) 296-317.

31. D. E. Akyol, and G. M. Bayhan, A review on evolution of production scheduling with neural Networks, Computers \& Industrial Engineering 53 (2007) 95-122.

32. J., Holland, Adaptation in Natural and Artificial Systems (University of Michigan Press, 1975, Ann Arbor).

33. C. Kahraman, O. Engin, I. Kaya, M.K. Yilmaz, An application of effective genetic algorithms for solving hybrid flow shop scheduling problems, International Journal of Computational Intelligence Systems, 1(2) (2008)134- 147.

34. DG. Dannenbring, An evaluation of fowshop sequencing heuristics. Management Science, 23 (1977) 174-82.

35. H. G, Campbell, R.A. Dudek, M.L. Smith, A heuristic algorithm for the n-job, m-machine sequencing problem. Management Science;16:B (1970) 630-637.

36. D.S. Palmer, Sequencing jobs through a multi-stage process in the minimum total time - a quick method of obtaining a near-optimum, Operational Research Quarterly, 16 (1) (1965) 101-107. 